\title{
Influence of Prior Thermomechanical Treatment on the Training Behaviour of a NiTi Alloy
}

\author{
D. Favier, Y. Liu* and P.Y. Manach**
}

Laboratoire Sols Solides Structures (3S) URA 1511 du CNRS, Université Joseph Fourier, BP. 53X, 38041 Grenoble cedex, France

* Departement of Mechanical and Materials Engineering, University of Western Australia, Nedlands, W.A. 6009, Australia

** Laboratoire de Génie Mécanique et Productique, Centre de Génie Industriel Guidel Plages, Université de Bretagne Occidentale, 56520 Guidel, France

\begin{abstract}
It is known that two-way memory effect (TWME) can be obtained in shape memory alloys by various types of thermomechanical cycling (training). Although much uncertainty still exists on the physical origin of the TWME, it is well recognised that dislocation arrays developed during training can have an important influence on the TWME. The investigation presented in this paper was designed to provide more information on the influence of initial dislocation structure on the TWME training behaviour of a near equiatomic NiTi alloy. Differences in initial dislocation structure were created by cycling specimens through austenite $\leftrightarrow$ martensite transformations in different modes: thermal cycling between $200 \mathrm{~K}$ and $370 \mathrm{~K}$, symmetric mechanical cycling to $\pm 5 \%$ shear strain, and combined thermal-mechanical cycling. Subsequent TWME training was performed in tension at a temperature just above Ms by deforming to $5 \%$ strain followed by heating. It is found that, whereas the deformation behaviour during training was different for specimens of different prior treatments, the final TWME after training was always the same.
\end{abstract}

\section{INTRODUCTION}

Shape memory alloys are well known to develop two-way memory effect after repeated thermomechanical cycling (training) such that a reversible shape change accompanies the austenite $\leftrightarrow$ martensite transformation in unstressed specimens. Although it is well recognised that dislocation arrays developed during training have an important influence on the TWME [1,2], the role of initial dislocation structure on the development of TWME during training is unknown. Experimental results giving information on these aspects are presented.

\section{EXPERIMENT PROCEDURE}

\subsection{Material}

A commercial NiTi shape memory alloy supplied by Cezus (France) of nominal composition Ti-50.2 at\% $\mathrm{Ni}$ was used in this study. It was supplied in the form of $0.5 \mathrm{~mm}$ sheet after a fabrication process consisting of several cold rolling steps with intermediate heat treatments at $975 \mathrm{~K}$ for $300 \mathrm{~s}$ in an argon atmosphere. The transformation behaviour of the as-received material as well as test specimens was determined using a Perkin-Elmer DSC 4 differential scanning calorimeter. DSC curves for an as-received specimen and a specimen thermally cycled for 100 cycles between $200 \mathrm{~K}$ and $370 \mathrm{~K}$ are shown in Fig. 1 . The as-received material showed a two-stage transformation during cooling and a one stage heating transformation cycle, ie. austenite $\leftrightarrow R$ phase $\leftrightarrow$ martensite on cooling and martensite $\leftrightarrow$ austenite on heating. 


\subsection{Prior treatment}

In order to create different initial dislocation structures, specimens for two-way memory training were prepared through different procedures prior to training. Sample A was in the as-received condition. Sample B was deformed in shear at $320 \mathrm{~K}$ on a shear device designed by P.Y. Manach [3,4]. At the temperature of test the specimen exhibited near-pseudoelastic behaviour and deformation occurred via the stress-induced martensitic transformation. The sample was cut from the as-received sheet into a rectangle of $30 \times 18 \mathrm{~mm}^{2}$ with a thickness of $0.5 \mathrm{~mm}$. The shear gauge zone was $30 \times 3 \mathrm{~mm}^{2}$. In order to avoid any training effect in the shear deformation, the sample was deformed symmetrically in two opposite shear directions, the deformation amplitude increasing progressively each cycle from 0 to $\pm 5 \%$, being kept constant for 10 cycles at $\pm 5 \%$, and then decreasing in steps down to zero (Fig. 2). Two other samples were prepared by thermal cycling. Thermal cycling was performed manually between two containers containing boiling water and methanol chilled with liquid nitrogen. 100 thermal cycles were performed on each of sample $C$, which was in the as-received condition, and sample $D$, which had been shear deformed. The DSC curves for specimens B and D were similar to those of specimens A and C, respectively.

\subsection{Training procedure}

Training was carried out on wire samples in tension on an 8035 Instron universal testing machine. The Instron equipment was equipped with a thermostatically controlled liquid bath so that the testing temperature could be varied between $260 \mathrm{~K}$ and $370 \mathrm{~K}$ with an accuracy of $\pm 0.5 \mathrm{~K}$. Deformation of specimen was evaluated from measurement of the crosshead displacement. Specimens were cut from the sheet material into $0.5 \times 0.5 \times 24 \mathrm{~mm}$ wires using a low speed diamond saw and then fabricated by swaging mild steel heads to the ends of the wires for gripping, leaving a gauge length of $16 \mathrm{~mm}$ [5]. For the specimen that had been previously deformed in shear, the wire was cut from the shear gauge zone. The training procedure consisted of 20 cycles, each cycle comprising three stages. The first stage was to cool the specimen from $370 \mathrm{~K}$ (>Af) to $285 \mathrm{~K}$ under no stress. In the second stage the specimen was deformed up to a total tensile strain of $5 \%$ isothermally at $285 \mathrm{~K}$, followed by unloading. The specimen was then heated back to $370 \mathrm{~K}$ after releasing the grips for free contraction, realising the one-way memory effect. The stress-strain curves of the first, tenth and twentieth training cycles are shown in Fig. 3 for a specimen in the as-received condition. It ought to be noted that the determination of the residual strain is not very accurate due to the method used to measure the deformation and the release of the grips between each training cycle.

\subsection{Measurement of the developed two-way memory effect.}

After training, the TWME was measured on a specially designed training device $[5,6]$, which enables the measurement of tensile strain during a thermal cycle with thermal expansion being compensated for. The two-way memory effect was measured between two extreme temperatures using boiling water and purging liquid nitrogen. The average of the values of the TWM strains on cooling $\left(\varepsilon_{\text {cool }}\right)$ and on heating $\left(\varepsilon_{\text {heal }}\right)$ was taken as the two-way memory strain $\left(\varepsilon_{\mathrm{tw}}\right)$.

\section{RESULTS.}

\subsection{Two-way memory effect}

Measurements of TWME of samples A, B, C and D are shown in table 1. For sample D two measurements were made, one after 20 training cycles and the other after 10 training cycles. It is seen that significant TWME was developed by training in all cases.

\subsection{Mechanical behaviour during training}

Although the final TWME is similar in all the samples, the mechanical behaviour during training is different. In supplementary to Fig. 3, selected stress-strain curves for the first, tenth and twentieth training cycles are shown in Fig. 4 for sample C. It is seen that for the as-received sample, training increased the transformation stress whereas for the thermally cycled sample $C$ the transformation stress remained nearly constant during training. The mechanical behaviours during training for samples $\mathrm{B}$ and $\mathrm{D}$ were similar to those of samples $\mathrm{A}$ and $\mathrm{C}$, respectively. To analyse the difference in mechanical behaviour of the four samples during training, stress-strain curves of the first and twentieth training cycles for the four samples are replotted in Figs. 5 and 6, respectively. It is seen that the initial stress for 
inducing martensitic transformation, as measured in the plateau region at $2 \%$ apparent strain, in the asreceived sample is $\sim 85 \mathrm{MPa}$ at the testing temperature. Mechanical cycling (sample B) did not change this stress. Thermal cycling, however, increased the initial transformation stress significantly. For the asreceived (Sample C) and mechanically cycled (Sample D) materials, thermal cycling for 100 cycles increased the plateau stress to $\sim 140 \mathrm{MPa}$ and $165 \mathrm{MPa}$, respectively (Fig.5). The final transformation stress in the training procedure, however, is surprisingly the same for all the samples, regardless of their prior thermomechanical treatments (Fig.6).

Table 1 : measurements of the developed two-way memory effect.

\begin{tabular}{|c|c|c|c|c|c|}
\cline { 2 - 6 } \multicolumn{1}{c|}{} & $\begin{array}{c}\text { Sample A } \\
\text { (as received) }\end{array}$ & $\begin{array}{c}\text { Sample B } \\
\text { (shear } \\
\text { deformed) }\end{array}$ & $\begin{array}{c}\text { Sample C } \\
\text { Thermally } \\
\text { cycled }\end{array}$ & $\begin{array}{c}\text { Sample D after } \\
\text { 20 sequences. }\end{array}$ & $\begin{array}{c}\text { Sample D after } \\
\text { 10 sequences. }\end{array}$ \\
\hline$\varepsilon_{\text {cool }}(\%)$ & 1.75 & 1.95 & 1.96 & 2.02 & 1.6 \\
\hline$\varepsilon_{\text {heat }}(\%)$ & 1.87 & 2.05 & 2.08 & 2.16 & 1.77 \\
\hline$\varepsilon_{\text {two-way }}(\%)$ & 1.81 & 2.00 & 2.02 & 2.09 & 1.68 \\
\hline
\end{tabular}

\section{DISCUSSION.}

\subsection{Effect of Prior Treatment on TWME}

Most of the published TWME training studies have been performed on samples with the same initial conditions to avoid confusion in the effort to characterise TWME behaviour and to optimise training parameters [7-9]. Comparison of TWME training behaviour between different initial microstructural conditions of given alloys has only been attempted with respect to cold working and annealing conditions [6]. It was shown that low temperature annealing after cold working leads to a poor training ability and a low level TWME in a binary near-equiatomic NiTi alloy. The maximum TWME obtained was associated with a fully recrystallised microstructure. The appearance of the $R$ phase transition prior to martensite on stress-free cooling was also found to have a determining effect on cancelling the development of a significant TWME by training $[6,10]$. However, similar training on a NiTiFe alloy showed that it was possible to develop a compatible TWME by training even when the $R$ transition existed (unpublished research). Thus, it was concluded that a high density of randomly distributed dislocations, resulting from cold working for instance, in the initial microstructure was detrimental to the development of TWME. More careful analyses on the relationship between the accumulated residual strain, which is an indication of the increase of dislocation density, and two-way memory strain in alloys capable of developing high level TWME during training showed that the optimum TWME was associated with a minimum residual strain and that most dislocations generated during training were undesirable for obtaining TWME [6,7].

This work is the first attempt to investigate into the role of the initial dislocation structure on the development of TWME by training. It is seen from Table 1 that prior thermomechanical treatment has no significant influence on the final TWME. This observation coincides with the suggestion that the magnitude of TWME is only determined by the number of training cycles and the magnitude of transformation strain induced by the training stress [5-9], although this suggestion was made on the basis of comparison of samples of the same conditions in the same training procedures. The present results, on the other hand, appear in contradiction with some other early conclusions, such as the detrimental effect of cold working and low temperature annealing on TWME [6]. Stalmans et al [7-9] suggested that thermal cycling diminished the maximum attainable TWME. This apparent disagreement with the conclusions of previous investigations may be rationalised as following.

Experiments reported in [6-9] were conducted under carefully selected testing conditions for obtaining optimum TWME. The maximum strain associated with the TWME was in the order of $>80 \%$ of the transformation strain induced by the training stress. Values of $\varepsilon_{\mathrm{tw}}$ lower than somewhat $60 \% \varepsilon_{\mathrm{r}}$ would be considered poor TWME. In the present work the TWME is $~ 50 \%$ of the transformation strain and would have not been classified as an optimum TWME. Thus the present result does not invalidate the previous suggestions that initial heavy dislocation structure cycling is detrimental to obtain an optimum TWME. 
The attainable maximum TWME for a given material has been found to depend on the training route [6]. Common training routes may be considered as the following basic types: pseudoelastic training (stressinduced martensitic transformation and stress-resisted recovery), shape memory training (stress-assisted martensite reorientation and free recovery) and constant load thermal cycling training. A modified constant load training route with the load removed during reversion was found most effective in developing maximum TWME [6], owing to its low tendency of generating excessive dislocations due to the low training stress and lack of resistance during reversion. Thus, various training routes may be characterised by their severity in terms of creating dislocations according to the means of inducing training strain, in the order of martensite reorientation - stress-assisted thermal transformation - stressinduced martensitic transformation from low to high. In this context the prior treatment may also be considered in terms of their severity in creating dislocations. The training route adopted in this study utilised the stress-induced martensitic transformation and it appeared sever enough to override the effect of the prior treatments, ie. the thermal cycling, shear deformation, incomplete annealing of the specimens, to impose a TWME. Should a shape memory training route have been employed a compatible TWME would have not been expected after training.

\subsection{Effect of Prior Treatment on Mechanical Behaviour}

Although the prior treatment imposed no influence on the TWME developed after the training process, it modified the mechanical behaviour of the material as observed during training. It is seen from Fig. 5 that the shear deformation did not alter the (initial) critical stress for inducing martensitic transformation. Thermal cycling, on the other hand, increased this stress to twice the value as before. Thermal cycling is well known to be capable of generating dislocations and modifying transformation behaviour [11,12], as evidenced by the changes in transformation calorimetric characteristics shown in Fig. 1. The increase in the (initial) critical stress for stress-induced martensitic transformation in these samples is believed to be associated with the decrease in martensitic temperature ; the constant testing temperature was effectively at a higher level relative to the Ms in these samples than in the as-received material. The decrease in Ms after thermal cycling was $\sim 10 \mathrm{~K}$ (Fig.1) and the increase in the critical stress was $\sim 75 \mathrm{MPa}$ giving a $\mathrm{d} \sigma / \mathrm{dT}$ value of $\sim 7.5 \mathrm{MPa} / \mathrm{K}$, which is consistant with previous measurements [5]. Shear deformation, on the other hand, appeared surprisingly less effective in introducing dislocations. This is probably because of the low magnitude of deformation, which may be easily accommodated by the martensitic transformation. In this context, the mechanical shear deformation adopted in this study is ranked less sever in damaging the matrix than thermal cycling.

Although the initial stresses for the martensitic transformation were different for different prior treatments, the final stresses after 20 training cycles were at the same level (Fig. 6). It has been observed that pseudoelastic cycling decreases the critical stress for martensitic transformation [11,12]. This is ascribed to the development of directional stress fields, which are also considered responsible for the TWME, during training. In this study the critical stress is found to increase with the number of training cycles in specimens $A$ and $B$, to remain relatively constant in specimen $C$ and even to decrease in specimen D. At the mean time, TWME developed. These observations and similar results obtained in shear training [4] post questions to the hypothesis of the development of directional stress fields and demands further investigation.

\section{CONCLUSION}

The present study may be concluded as following:

1) Similar TWME has been developed by the same training procedure in samples with different prior thermomechanical histories. The influence of a prior treatment on the final TWME is dependent on the training method as well as the treatment itself. In particular, the influence of a prior treatment is strong for a training route of low severity, as characterised by the ability to modify dislocation structure. It is suggested that pseudoelastic training, constant load training and shape memory training (as defined in this text) have in this order decreasing severities.

2) Mechanical shear deformation within the transformation strain range is less effective in causing permanent modification to microstructure than thermal cycling; thus subsequent thermal and mechanical behaviour is less affected. 
3) For the as-received material, an increase in the critical stress for stress-induced martensitic transformation is observed during mechanical-thermal cycling used to develop the TWME. This observation appears in contradiction to the hypothesis on the development of directional stress fields during stress-induced martensitic transformation. The uncertainty on this matter is to be clarified.

\section{Acknowledgments}

This work was performed during the stay of one of the authors (D.F.) at the University of Western Australia as a Gledden Senior Visiting Fellow. Support from the Gledden Foundation is gratefully acknowledged. The authors also wish to thank Prof.P.G.Mc Cormick for his help both in experiment and discussion.

\section{References}

[1] Perkins J., Sponholz R.O., Met.Trans., 15A, (1984), 313.

[2] Rios-Jara D., Guénin G., Acta Metall Mater., Vol 35, N.7, (1987), 109-

[3] Manach P.Y., Ph.D Thesis, Institut National Polytechnique de Grenoble, France, (1993).

[4] Manach P.Y., Favier D., Scripta Metall.Mater., Vol 28, (1993), 1417-1421.

[5] Liu Yinong, Ph.D Thesis, University of Western Australia, Australia, (1990).

[6] Liu Yinong, McCormick P.G., Acta Metall.Mater., Vol 38, N.7, (1990), 1321-1326.

[7] Stalmans R., Van Humbeeck and Delaey L., Acta Metall.Mater., Vol 40, N.3, (1992), 501-511.

[8] Stalmans R., Van Humbeeck and Delaey L., Acta Metall Mater., Vol 40, N.11, (1992), 2921-2931.

[9] Stalmans R., Van Humbeeck and Delaey L., Journal de Physique, C4, Vol.1, (1991), 403-408.

[10] Liu Yinong and McCormick, P.G., Scripta Metall.Mater, Vol.22, (1988), 1327-1330.

[11] Miyazaki, S., Eng. Aspects of Shape Memory Alloys, Butterworth-Heinemann, London, (1990), 394

[12] McCormick, P.G. and Liu Yinong, Acta Metall. Mater., 42, (1994), 2407-2413.

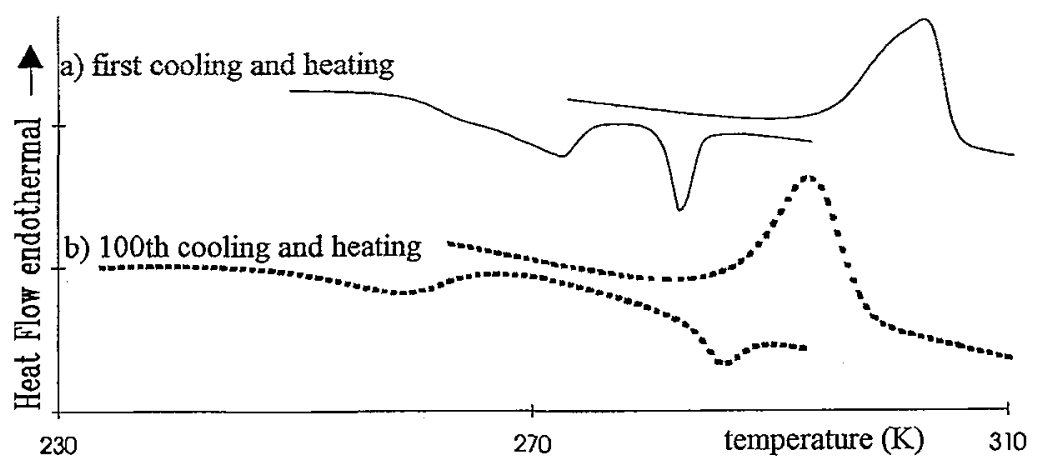

Figure 1 : DSC curves for as received material and after thermal cycles.
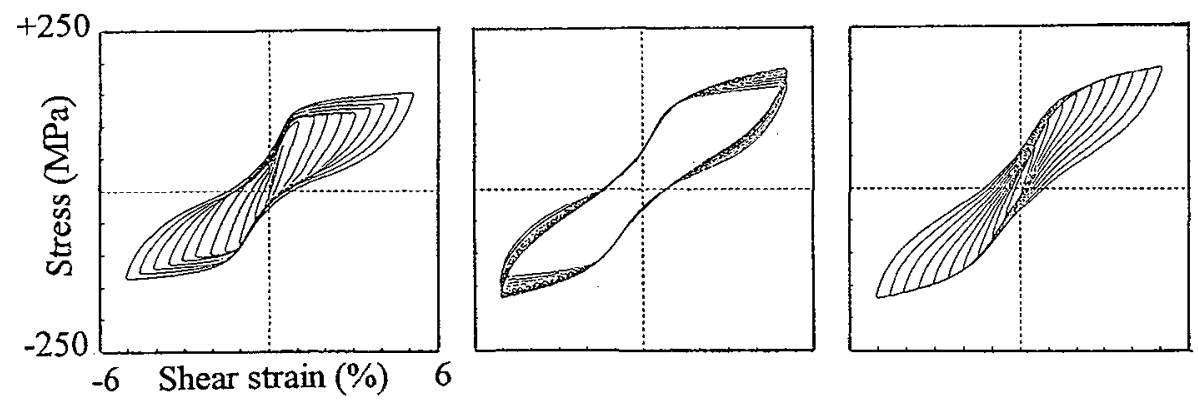

Figure 2 : Prior shear deformation. 

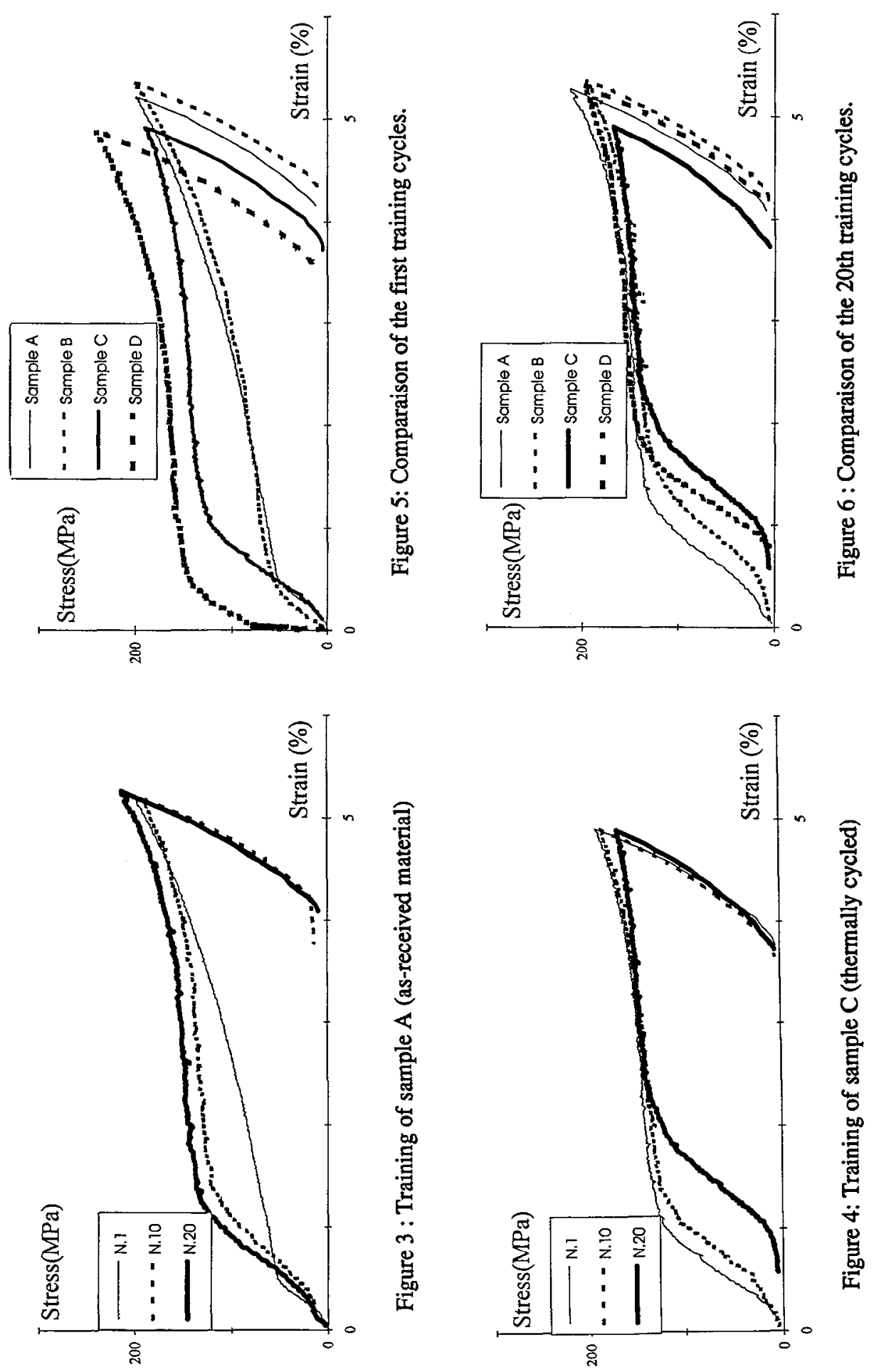